\title{
New Approach based on Simplification and partially fixing of Problem to solve Large Scale Vehicle Routing Problem
}

\author{
Shinya Watanabe ${ }^{1}$ Tetsuya Sato $^{2} \quad$ Kazutoshi Sakakibara $^{3}$ \\ ${ }^{1}$ College of Information and Systems,Muroran Institute of Technology, Japan, sin@csse.muroran-it.ac.jp \\ ${ }^{2}$ Mizuho Information \& Research Institute, Inc., Japan, tet su . en . sato@gmai l . com \\ ${ }^{3}$ Department of Information Systems Engineering, Toyama Prefectural University, Japan, \\ sakakibara@pu-toyama.ac.jp
}

\begin{abstract}
This paper presents a specialized evolutionary approach for large scale vehicle routing problems (VRPs). Our approach includes two original mechanisms; simplification of problem and partially fixing of customers' sequence. The first one tries to simplify the problem by integrating some neighbor customers into one group recursively and to iterate to restore the simplified problem to original one gradually. And second mechanism is to reduce the search space of the problem by fixing a part of customers' sequence. Our approach is designed for an effective search in large scale VRPs by the interaction of these mechanisms. Through applying the proposed approach to some test problems having different characteristics, the effectiveness of our approach is determined in comparison with normal approach (without our these original mechanisms).
\end{abstract}

Keywords: vehicle routing problem, large scale problem, evolutionary multi-criterion optimization

\section{Introduction}

Vehicle Routing Problems (VRPs) are well known as NP-hard combinatorial optimization problems arising in many distribution and transportation systems, such as postal delivery, school bus routing, newspaper distribution, etc. VRPs have attracted a great deal of attention since 1970's due to their wide applicability and economic importance(Braysy and Gendreau, 2005; Watanabe and Sakakibara, 2007).

Although the objective of most VRPs' application is to minimize the total area distance, VRPs inherently have multi-objective aspects such as the number of vehicles or the degree of dispersion between the distances of each vehicle. Therefore, there have been many studies using evolutionary multi-criterion optimization (EMO) algorithm to optimize multi-objective VRPs (Watanabe and Sakakibara, 2007; Jozefowiez et al., 2002).

Recently, data size and problem size has become larger scale according to technical advantages of storage performance and cloud technology. Since this trend causes new formidable issues such as the combinatorial explosion and the increment in computational cost, previous approaches are difficult to obtain solutions to fill required quality in real time.

In this research, we propose a new approach dedicated to very large scale VRPs. The proposed approach has two distinguishing mechanisms; simplification of problem and partially fixing of customers' sequence. The first mechanism tries to obtain high quality candidate solutions at early stage by mixing simplification and gradual restoration techniques. In particular, simplification is used firstly to reduce the number of customers apparently by gathering some neighborhood customers to one virtual customer.

Also, the second fixing mechanism is used to reduce search space by fixing a part of customers' sequence and perform an efficient refinement of the obtained solution by applying the first mechanism. The fixing of customers' sequence is very reasonable way to reduce a number of combination in large scale problem.

Through some test examples of Cordeau's instances from VRP website $^{1}$, we showed that the proposed approach can obtain high quality solution in the case of very large problem size.

\section{Vehicle Routing Problem}

There are many different types in vehicle routing problems (VRPs) based on the type of handling constraints. This paper deals with the multiple depots vehicle routing problems (MDVRPs) (Luo et al., 2013) having only a capacity constrain, which is generally called the capacitated VRPs (CVRPs).

The definitions of CVRPs and MDVRPs can be described as below.

\subsection{Capacitated Vehicle Routing Problems}

There are many kinds of VRPs according to the type of constraints. CVRPs having only a capacity constrain can be defined as follows (Braysy and Gendreau, 2005):

- All vehicles start from the depot and visit the assigned customer points, then return to the depot. Here, a route is formed by the sequence of the depot

\footnotetext{
${ }^{1}$ VRPwebsite http: //www . bernabe.dorronsoro.es/vrp/.
} 
and the customer points visited by a vehicle. Therefore the number of vehicles is same as the number of route. Moreover, each customer is visited only once by exactly one vehicle.

- Each customer asks for a weight $w_{i}(i=1, \ldots, N)^{2}$ of goods and a vehicle of capacity $W$ is available to deliver the goods. In this paper, we used the same capacity $W$ for all vehicles.

- A solution of the CVRPs is a collection of routes where the total route demand is at most $W$.

In this paper, we treated CVRPs having multiple depots (MDVRPs). In MDVRPs, departure and arrival points of each vehicle should be same.

VRPs have a number of objectives, such as minimization of the total travel distance, minimization of the number of routes, minimization of the duration of the routes, etc. In this paper, we treated VRPs as two objective problem; minimization of the total travel distance $\left(F_{\text {dist }}\right)$ and the variance of travel distance of each vehicle $F_{\mathrm{var}}$. The formulas of these objectives are as follows:

$$
\begin{aligned}
& \operatorname{minimize} F_{\mathrm{dist}}=\sum_{m=1}^{M} c^{m} \\
& \text { minimize } F_{\mathrm{var}}=\frac{1}{M} \sum_{m=1}^{M}\left(\bar{c}-c^{m}\right)^{2}
\end{aligned}
$$

where $M$ is the total number of routes and $c^{m}$ and $\bar{c}$ indicate $m$ th route distance and the average value of $c^{m}$, respectively.

\subsection{Multi-Depot Vehicle Routing Problems}

MDVRPs add one more constraint to CVRPs relative to multi-depots. This constraint is that starting point and ending point of each vehicle should be the same depot.

\section{Proposed approach}

In this paper, we proposed a new approach specialized for large scale VPRs. The main feature of the proposed approach is to have two distinguishing mechanisms; simplification and fixing mechanisms. The first one is used for obtaining high quality solutions at early search phase and the second one is assumed to perform an efficient refinement of solutions at late search phase.

In this section, the details of the proposed approach were explained.

\subsection{The flow of the proposed approach}

Here, we described the detail of our approach as follows.

Step 0: The setting of initial parameters

- $N$ The number of initial population

- $G_{S}$ The upper period of the search stagnation (the timing parameter of dissolving cluster)

\footnotetext{
${ }^{2} N$ is the number of customers.
}

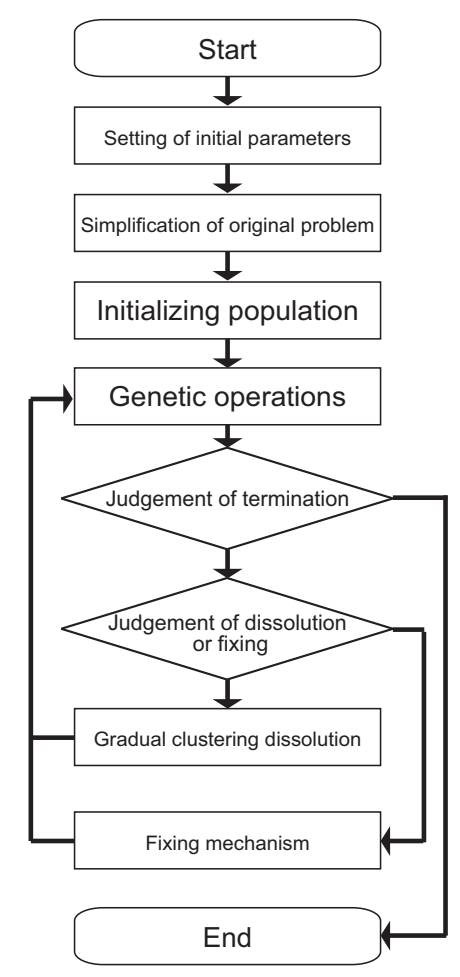

Figure 1. Flowchart of the proposed approach.

- $G_{R}$ The upper period of the search stagnation (the timing parameter of fixing mechnism)

- $E_{\text {Final }}$ Terminal criteria

Step 1: Simplification of the problem

In order to simplify the original problem, neighborhood customers would be gathered to one virtual customer by clustering method and this gathering process would be repeated until the number of customer reaches the pre-defined number.

Step 2: Initializing population

Create $N$ initial individuals and set the parameter $t$ representing the generation $t=0$ and the parameter $g_{s}$ representing the term of search stagnation $g_{s}=0$.

Step 3: Updating solutions

Solutions would be improved by genetic operations. And the solution having the best value of $F_{\text {sum }}{ }_{T}^{\mathrm{T}}$ at $T$ generation would be found. If $F_{\text {best }}{ }^{\mathrm{T}-1}$ and $F_{\text {best }}^{T}$ are same, the value of $g_{s}$ is incremented by one $\left(g_{s}=\right.$ $\left.g_{s}+1\right)$ and in other case $\left(F_{\text {best }}^{T-1} \neq F_{\text {best }}^{T}\right)$, reset the value of $g_{s}\left(g_{s}=0\right)$. However $g_{s}$ would be kept 0 until this step repeat $G_{r}$ times.

Step 4: Judgment of termination

If termination condition is satisfied, terminate this optimization process. Also, if $G_{S} \leq g_{s}$ and $p \neq 1$, go to Step 5 (clustering dissolution phase), and in the case of $G_{R} \leq g_{s}, p=1$, go to Step 6 (fixing mechinsm). Otherwise, go back to Step3.

Step 5: Cluster dissolution

In order to reinstate simplified problem, gradual dis- 
solution mechanism would dissolve clustering in a limited area (the details of this mechanism is described below). After resetting $g_{s}=0$, go back to Step4.

Step 6: Fixing and unfixing of customers' sequence

In order to reduce search space by fixing a part of customers' sequence, fixing mechanism would implement the fixing or unfixing of customers' sequence(the details of this mechanism is described below). After resetting $g_{s}=0$, go back to Step4.

Hereinafter, simplification of problem of of Step1, gradual cluster dissolution mechanism of Step 5 and fixing mechanism of Step 6 are described in detail.

\subsection{Simplification of the problem}

Our simplification technique tries to reduce the number of customers apparently by gathering some neighborhood customers to one virtual customer. In this simplification, clustering method is used to group a set of customers and some unique features in VRPs are considered.

As a results of analyzing best known solutions of some different famous CVRP instances, we could find the relation between depots and customers. The customers near depot tend to belong different routes even though they are very close. On the other hand, in the case of the customers being far from depot, customers in the near distance tend to belong same route.

Therefore we designed our simplification that the customers being father away from depots result in a higher rate of grouping customers (creating sets).

Also, in this simplification, we restrict grouping customers in case that their distance is over a certain amount and the total weight of grouping customers is over a weight capacity of vehicle.

The flow of this simplification is shown as below.

Step 0: Creating initial clusters

Creating initial clusters $C_{i}(i=1, \ldots, N)$ having each one customer and setting the initial clusters' total load $w^{C_{i}}(i=1, \cdots, N)$. Here, set the number of customer in each cluster $P_{i}=1$ and current total number of clusters $N^{C}=N$.

Step 1: Calculating the maximum number of customers for each cluster

Calculating the maximum number of customers $P_{i}^{\max }$ for each cluster. This $P_{i}^{\max }$ is defined according to the distance between cluster and the depot. We set larger $P_{i}^{\max }$ of cluster larger distance from depot.

Step 2: Selecting the target cluster

Selecting the target cluster satisfying below equation (1) from unselected clusters.

$$
\max _{i=1, \cdots, N C} d_{\left(C_{i}, C_{0}\right)}
$$

where $C_{0}$ is depot and $d_{\left(C_{i}, C_{j}\right)}$ indicates the shortest distance between two clusters. If there are no clus- ters which satisfy the above equation, finish this procedure.

Step 3: Judgment of termination

If $N^{C} \leq N^{P}$, finish this procedure. Otherwise, go to next step.

Step 4: Finding set of clusters to combine

Selecting set of clusters to combine. Here, $C_{i}$ and $C_{j}$ satisfying below equation are selected.

$$
\begin{array}{ll}
D^{T} & \geq \min _{j=1, \cdots, N^{C} \mid j \neq i} d_{\left(C_{i}, C_{j}\right)} \\
\text { s.t. } & W \geq w^{C_{i}}+w^{C_{j}} \\
& \left(j=1, \cdots, N^{C} \mid i \neq j\right) \\
& P_{i}^{\max } \geq P_{i}+P_{j} \\
& \left(j=1, \cdots, N^{C} \mid i \neq j\right)
\end{array}
$$

where $D^{T}$ is the upper limit distance of combing clusters. Also, $W$ indicates the upper load capacity and $d_{\left(C_{i}, C_{j}\right)}$ represents the distance between two clusters.

Step 5: Combination of two clusters

Combining two clusters $\left(C_{i}\right.$ and $\left.C_{j}\right)$ and updating cluster's total load and the number of customer within cluster. Specifically, the information of $C_{j}$ is integrated into that of $C_{i}$ and remove $C_{j}$. After renewing $N^{C}=N^{C}-1$, go back to Step 3 .

As mentioned above, this simplification tries to combine two adjacent clusters into one until the total number of clusters reaches the predefined number.

\subsection{Gradual cluster dissolution}

This technique gradually restore simplified problem to original one. If the simplified problem is restored at once, the differences between before and after problems is so large that the solutions of prior problem wouldn't contribute to the search in a new integrated problem. Therefore, we try to control the differences between before and after problems small by gradually restoration. In the case that the difference of two problems is small, the solutions of prior problem would be good seed solutions in antecedent one and this would tend toward an effective search.

There two key points in this dissolution; the timing of dissolving cluster and the determination which cluster is dissolved next. For former point, we use the term of search stagnation as the timing of dissolving cluster. Specific conditions of dissolution timing are that a best incumbent solution having best $F_{\text {sum }}$ value remains unchanged for a certain predefined generation $\left(G_{S}\right)$.

On the other hand, we specify the region for dissolving cluster in order to perform locally-concentrated search regarding the determination of dissolving cluster. Specifically, we define $\Theta$ angle region around on depot for the dissolution region and dissolve clusters within this angle region. Also, we define the search region having twice the 
area of dissolution region and restrict the range of search in order to increase the search effectiveness.

The details of this dissolution is described as below.

Step 1: Judgment for restoring simplified problem to original one

If current generation $g$ satisfied $g \geq G_{F}$, dissolve every cluster in order to return to original problem and finish this flow.

Step 2: Timing judgement for partially dissolution

If the above two conditions about dissolution timing were satisfied, go to Step 3. Otherwise, finish this flow.

Step 3: Determination of dissolution area for dissolving cluster

Calculate the dissolution area utilizing unit vector $u=(1.0,0.0)$, depot and $0 \leq \theta \leq 360$.

Step 4: Dissolving cluster within dissolution area

Dissolve every cluster having multiple customers within dissolution area.

\subsection{Fixing mechanism}

This fixing mechanism has two opposite mode; fixing and unfixing mode and it depends on the condition which mode is performed if this mechanism is called. In particular, we use the ratio of the total number of customers as the switching condition. In the case this ratio is under 0.8 , fixing mode gets executed, otherwise, unfixing mode is carried out.

In fixing mode, a couple of customer that are the highest potential as customer's sequence is fixed. Since the fixing customers are excluded from search space, we expect that this fixing would promote the efficiency of search and reduce a waste of search.

Of course, this fixing has a risk to fix a wrong couple of customers and this wrong fixing may cause to be trapped in local optima. Due to reduce this risk to the absolute minimum, the following function is used to select a couple of customers as a fixing sequence.

$$
F_{\text {bond }}=\frac{d_{\left(C_{i}^{k}, C_{i+1}^{k}\right)}}{d_{\left(0, M_{\left(C_{i}^{k}, C_{i+1}^{k}\right)}\right)} \times 2}
$$

where $d_{(a, b)}$ represents the distance between $a-b, C_{i}^{k}$ indicates the customer that is $i$ th visited in route $k, 0$ is depot and $M_{(a, b)}$ means the middle point between $a-b$.

(5) consists of two parts; the distance from the middle point and the distance from the depot. Since a lower value of (5) means more appropriate for fixing a couple of customers, the couple having lowest value of (5) is selected.

On the other hand, unfixing mode tries to release the fixing customers' sequence in the limited area. The basic idea of unfixing is the same as cluster dissolution in section3.3. Therefore, this mode unfixes every fixing customers within the area calculated the same as cluster dissolution technique. We expects this unfixing has the effect
Table 1. Instances.

\begin{tabular}{c||c|c|c|c}
\hline Instance & tai385 & $\begin{array}{c}\text { triple- } \\
\text { tai385-d3 }\end{array}$ & $\begin{array}{c}\text { sixth- } \\
\text { tai385-d1 }\end{array}$ & $\begin{array}{c}\text { sixth- } \\
\text { tai385-d6 }\end{array}$ \\
\hline \hline \# customers & 385 & 1155 & 2310 & 2310 \\
\hline \# depots & 1 & 3 & 1 & 6 \\
\hline
\end{tabular}

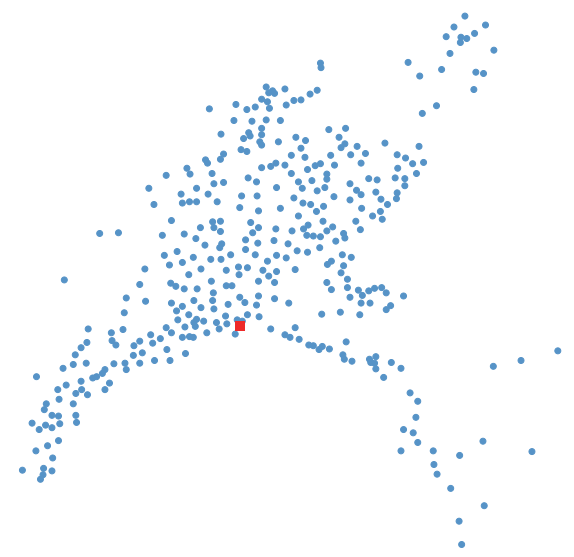

Figure 2. The distribution of tai385.

of reducing the risk caused by a wrong fixing customers' sequence.

\section{Numerical Examples}

We used MOEA/D(A Multiobjective Evolutionary Algorithm Based on Decomposition)(Zhang and Li, 2007) as EMO algorithm. And we treated this MVRPs as two objective problem having $F_{\text {dist }}$ and $F_{\text {var }}$ of expression (1) and (2) respectively. Table 2 shows parameters we used. As shown in Table 2, the results of this section were of 10 trials.

In this experiment, we investigated the characteristics and effectiveness of the proposed approach by comparing the performance of the approach without our original mechanisms(normal method). In particular, we used four cases for comparison; normal (without every mechanisms), simplification (with simplification and without fixing), immobilization (without simplification and with fixing) and proposed (with every mechanisms) methods.

\subsection{VRPs Instances}

We used four test problems based on Taillard's instances (tai385) from VRP website;tai385, triple-tai385-d3, sixthtai385-d1 and sixth-tai385-d6. tai385 is original Taillard's instances and triple-tai385-d3 is created by the combination of 3 tai385. Although sixth-tai385-d1 and sixthtai385-d6 are composed of 6 tai385, the former instance has only one depot and the latter has 6 depots, respectively. 
Table 2. Used Parameters.

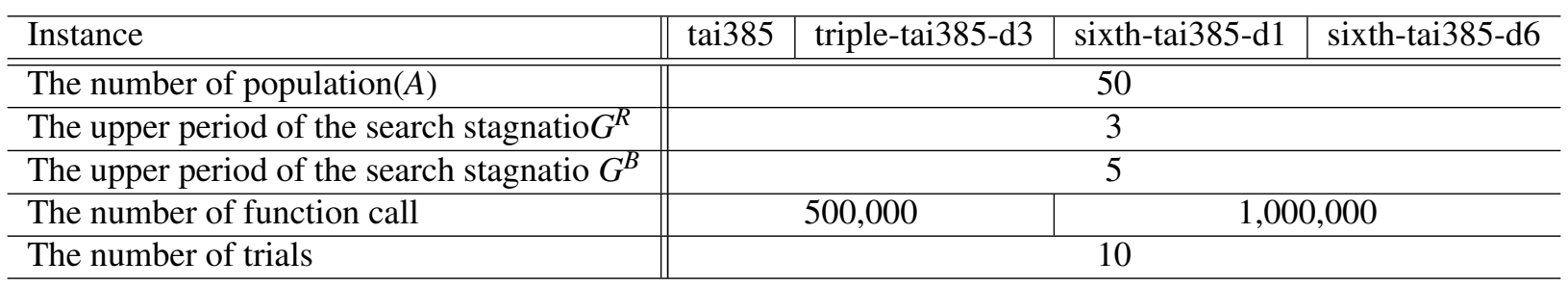

Table 3. Computational times (average).

\begin{tabular}{c||r|r|r|r}
\hline Problem & Normal & Simplification & Immobilization & Proposed \\
\hline \hline tai385 & $23 \min 32 \mathrm{~s}$ & $22 \min 30 \mathrm{~s}$ & $11 \min 3 \mathrm{~s}$ & $10 \min 11 \mathrm{~s}$ \\
\hline triple-tai385-d3 & $12 \min 25 \mathrm{~s}$ & $12 \min 36 \mathrm{~s}$ & $7 \min 7 \mathrm{~s}$ & $9 \min 12 \mathrm{~s}$ \\
\hline sixth-tai385-d1 & $6 \mathrm{~h} 22 \min 46 \mathrm{~s}$ & $5 \mathrm{~h} 11 \min 11 \mathrm{~s}$ & $5 \mathrm{~h} 15 \min 25 \mathrm{~s}$ & $5 \mathrm{~h} 12 \min 59 \mathrm{~s}$ \\
\hline sixth-tai385-d6 & $1 \mathrm{~h} 12 \min 54 \mathrm{~s}$ & $55 \min 50 \mathrm{~s}$ & $42 \min 46 \mathrm{~s}$ & $42 \min 42 \mathrm{~s}$ \\
\hline
\end{tabular}

Table 4. The values of hyper volume.

\begin{tabular}{c||r|r|r|r}
\hline Problem & Normal & Simplification & Immobilization & Proposed \\
\hline \hline tai385 & $8.15 \times 10^{7}$ & $8.38 \times 10^{7}$ & $7.95 \times 10^{7}$ & $8.22 \times 10^{7}$ \\
\hline triple-tai385-d3 & $6.44 \times 10^{7}$ & $6.86 \times 10^{7}$ & $5.44 \times 10^{7}$ & $5.66 \times 10^{7}$ \\
\hline sixth-tai385-d1 & $1.96 \times 10^{9}$ & $2.62 \times 10^{9}$ & $1.72 \times 10^{9}$ & $1.86 \times 10^{9}$ \\
\hline sixth-tai385-d6 & $2.96 \times 10^{8}$ & $6.73 \times 10^{8}$ & $2.13 \times 10^{8}$ & $6.12 \times 10^{8}$ \\
\hline
\end{tabular}

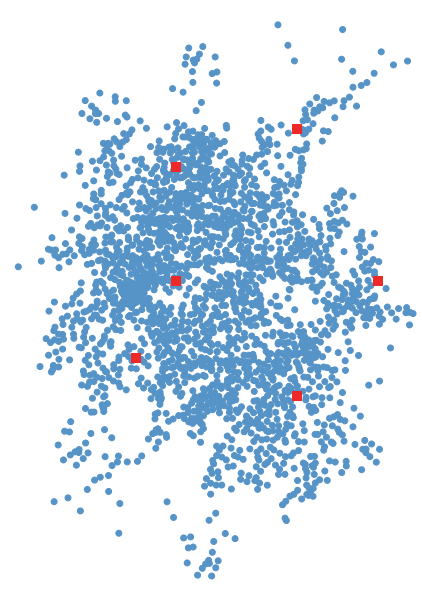

Figure 3. The distribution of sixth-tai385-d6.

The details of four test problems is shown in Table 1 and the distribution of customers in tai385 and sixth-tai385-d6 are shown in Figure 2 and Figure 3.

\subsection{Performance measures}

In this experience, we used two different type of performance measures for evaluating the obtained solutions; hyper volume (HV) (Zitzler and Thiele, 1998). HV can be calculated as the volume covered by non-dominated solutions and can be treated as an overall measure. In HV, the higher values mean the better solutions.

\subsection{Results and Analysis}

The results of performance measures are shown in Table 4. The transitions of minimum $F_{\text {dist }}$ value in each problem are shown in Figure 4.

From Table 4, we could find that simplification case could get better solutions than those of the other cases in totally. Proposed method obtained better results in sixthtai385-d6, but in other cases the results of normal method were better than those of proposed method.

However, Figure 4 indicated a bit different result. From these cases, simplification and proposed methods could obtain the solution with better quality. Particularly, in the problem having large customer problems the performance of these methods were overwhelming. Also, in the case of small size problem, normal method were superior to our methods.

From these result, every mechanisms in proposed method have an advantage in large scale problem and are not efficient in the small size problem. Also, simplification could indicate better results in every large scale problem but the performance of immobilization were low in totally. This fact indicates that the only fixing mechanism is difficult to improve a search performance.

On the other hand, Table 3 indicated that every our methods could finish at shorter times than that of normal method. This means every mechanisms of proposed method has an effect on accelerating the search process. Especially, this tendency were significant in large scale problems. 


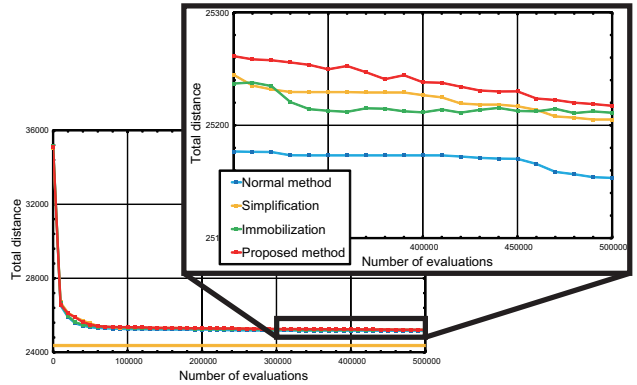

(a) tai385 (\#depots = 1)

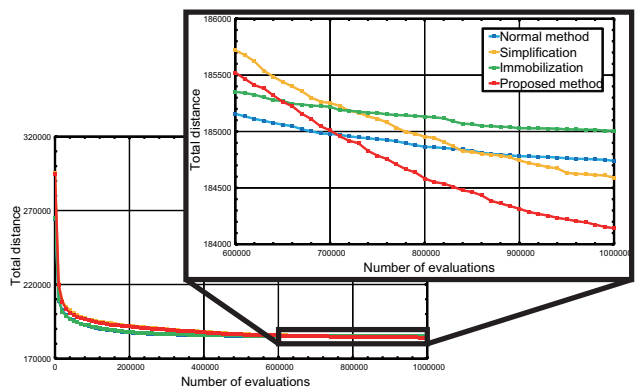

(c) sitxth-tai385-d1 (\#depots = 1)

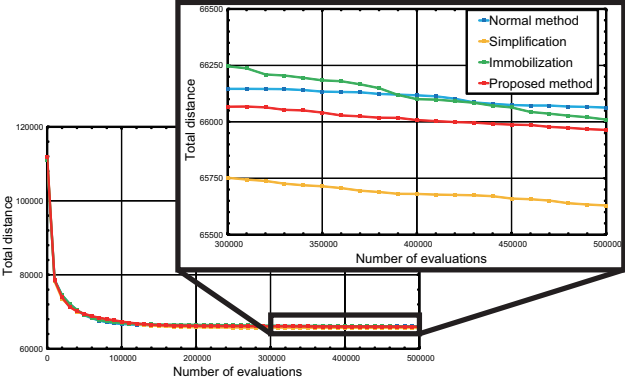

(b) triple-tai385-d3 (\#depots = 3)

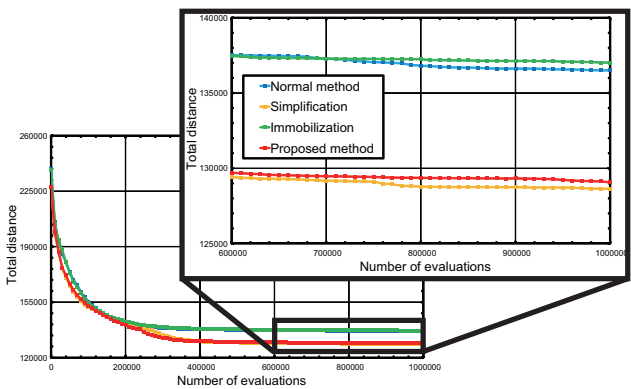

(d) sixth-tai385-d6 (\#depots = 6)

Figure 4. The transitions of minimum $F_{\text {dist }}$ value.

\section{Conclusions}

In this paper, we proposed a new method based on simplification and fixing mechanisms for large scale vehicle routing problems (VRPs). The first mechanism is aimed to obtain high-quality solutions at early stage by mixing simplification and gradual restoration techniques. And the second mechanism is to refine the obtained solution through applying the first mechanism more efficiently due to a reduction of search space.

We investigated the effectiveness of the proposed method and our mechanisms in the proposed method by comparison of its performance with the cases having no our mechanisms.

Numerical experiments clarified the following points:

1) Our mechanisms could obtain the solution with better quality in large customer problems, but in the case of small size problem the proposed method and our mechanisms were not good.

2) To use only fixing mechanism is not effective way for improving a search performance.

As future works, we would investigate the influence of clustering in more detail and try to apply the proposed approach to another very large scale VRPs. Also, we would advance the application to more practical problems (including real world problems).

\section{Acknowledgment}

This research was funded in part by a Grant-in-Aid for JSPS fellows (No. 26330269). Also, this work is partially supported by "Joint Usage/Research Center for
Interdisciplinary Large-scale Information Infrastructures (jh160047)" and "Information Initiative Center, Hokkaido University (A2-1)" in Japan.

\section{References}

O. Braysy and M. Gendreau. Vehicle routing problem with time windows, part i: Route construction and local search algorithms. Transportation Science, 39(1):104-118, 2005.

N. Jozefowiez, F. Semet, and E. Talbi. Parallel and Hybrid Models for Multi-objective Optimization: Application to the Vehicle Routing Problem. In Parallel Problem Solving from Nature-PPSN VII, pages 271-280, 2002.

J. Luo, X. Li, and M. Chen. Multi-phase meta-heuristic for multi-depots vehicle routing problem. Software Engineering and Applications, pages 82-86, 2013.

S. Watanabe and K. Sakakibara. A multiobjectivization approach for vehicle routing problems. In Evolutionary Multi-Criterion Optimization. Fourth International Conference (EMO 2007),Lecture Notes in Computer Science., volume 4403, pages 660-672, 2007.

Q. Zhang and H. Li. Moea/d: A multiobjective evolutionary algorithm based on decomposition. IEEE Trans. Evolutionary Computation, 11(6):712-731, 2007.

E. Zitzler and L. Thiele. Multiobjective Optimization Using Evolutionary Algorithms - A Comparative Case Study. Parallel Problem Solving from Nature - PPSN-V, pages 292-301, 1998. 